\title{
Microfluidics as an Emerging Platform for Tackling Antimicrobial Resistance (AMR): A Review
}

\author{
Sammer-ul Hassan ${ }^{\mathrm{a}, \mathrm{b},}$, Xunli Zhanga,b,*
}

${ }^{a}$ Bioengineering Research Group, Faculty of Engineering and Physical Sciences, University of Southampton, Southampton SO17 1BJ, UK; ${ }^{b}$ Institute for Life Sciences, University of Southampton, Southampton SOI7 1BJ, UK

\begin{abstract}
:
Antimicrobial resistant bacteria have been recognised as a global threat and require a robust and collective response from every stakeholder of the society and public health institutions. Currently available technologies to tackle antimicrobial resistance (AMR) have been found to be time-consuming, expensive, labour intensive and central labbased which poses increasing challenges, especially in remote areas where resources are limited. In contrast to most of the conventional technologies, microfluidics has become an enabling platform for AMR testing, allowing simple, robust, cost-effective and

Dr S Hassan

Prof X Zhang portable diagnostics. Various microfluidics techniques have been developed and tested for bacterial identification and determination of minimum inhibitory concentration (MIC), demonstrating a broader understanding of the behaviour of antimicrobial resistant strains, and development of antimicrobial susceptibility (AST) tools. Miniaturised microfluidic systems have led to the integration of various tools on a single platform such as sample handling, controlled reactions and on-chip detections towards portable, simple, easy to use and POC diagnostics in clinical environments. However, the benefits of microfluidics technology have not been fully exploited in tackling AMR because current microfluidic-based studies provide valuable information about the antibiotic resistance but are far from deployment in clinical settings. Hence, there is a lack of reliable and accurate systems in the market. AMR is an ever-growing threat and microfluidics must act faster to provide commercially viable techniques to combat resistant bacteria and guide clinical decisions. This article reviews the current microfluidic-based technologies for rapid AST testing and future prospects of the microfluidics technology for tackling AMR in clinical settings.
\end{abstract}

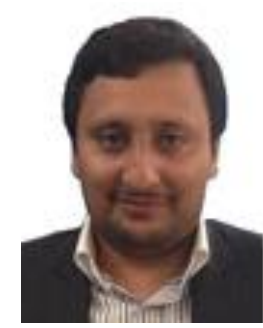

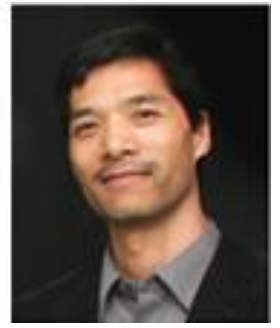

Keywords: Microfluidics, Point-of-care, Antimicrobial resistance (AMR), Lab-on-a-chip, Antibiotic, Capillary flow, Colorimetry, antimicrobial susceptibility testing (AST), minimum inhibitory concentration (MIC)

\section{INTRODUCTION}

Antimicrobial resistance (AMR) occurs when microbes become resistant to antibiotics causing complications and limited treatment options. AMR is more significant where antibiotics use is excessive or abusive, and the strains of bacteria grow resistant to antibiotic treatments. This is associated with hundreds of thousands of deaths every year (700,000 annually) while more and more bacteria are becoming resistant every day which could even lead regular injuries, infections or surgeries to death [1]. Global healthcare largely relies on the performance of antibiotics to tackle diseases and perform operations including cancer and organ transplantation. Therefore, failure of antibiotics would lead to less effective therapies and more fatalities [2].

Excessive and unnecessary use of antibiotics is regarded as the most significant cause of bacterial resistance as broadspectrum drugs are administered for common infections. Another practice is that the drugs are not monitored at hospitals and given to patients for home use which is

\footnotetext{
*Address correspondence to this author at the Department of Mechanical Engineering, Faculty of Engineering and Physical Sciences, University of Southampton, Southampton, UK; Tel: ++44-238-059-5099;

E-mails: XL.Zhang@soton.ac; S.Hassan@soton.ac.uk
}

unavoidable because of the excessive costs associated with patient monitoring at hospitals. Additionally, an alarming situation is that in developing countries antibiotics can be purchased over the counter without prescriptions and used for common infections or illnesses that affect body's immunity against bacterial infections. Among the most quantitatively used antibiotics sectors is the use of drugs in animal farms to promote growth weight and prevention of infections and diseases. This subsequently results in the spread of AMR as bacteria raised in farms end up in the human body and the environment [3]. The supply and demand issue of new antibiotics has also led to an increase in fatalities due to the microbes becoming resistant to the current drugs [1]. Therefore, a social and public awareness and toughened regulations to combat AMR must be increased globally.

Current technologies for bacteria and its resistant strains identification and antimicrobial susceptibility testing (AST) are mostly central-lab based in hospitals, which generally take days to weeks to get results. These tools and procedures are expensive, laborious and skills based. AST provides essential information about susceptibility of the antibiotics for guiding the infection treatments. The standard AST methods commonly used are disc diffusion for detection of bacterial 
strains resistivity towards antibiotics or broth dilution for determination of minimum inhibitory concentrations (MIC). These methods usually take 3-7 days for completion of results and include longer culture and incubation times, large amounts of samples and antibiotics, the possibility of false positives and sophisticated arrangements to interpret results $[4,5]$. Several other techniques have been developed to address these shortcomings such as PCR based resistant gene detection, mass spectrometry, genome sequencing and microbeads technology [6-10]. However, these upgrades are not sufficient to provide the full benefits required for rapid, cost-effective and reliable ASTs. Henceforth, a collective global and befitting response is necessary to tackle the everincreasing AMR effect on healthcare systems and societies. This goal can be achieved by limiting prescription of antibiotics until necessary, developing new antibiotics for specific diseases at speed much higher than current supply [1], and developing inexpensive, robust and near-patient portable systems for faster identification of microbes, AST testing and MIC determinations.

There is an ever-increasing demand for developing point-ofcare (POC) diagnostics tools for rapid and near patient AMR testing. Microfluidics, an essential and fundamental technique to develop POC devices, is referred to as a flow of minute amount of liquids in microchannels (micrometre dimensions). Fundamental to the development of POC diagnostics has been the use of microfluidics, which offers small sample volume consumption and the combination of multiple sampleprocessing steps into a single device. Microfluidics has been a critical technology for over two decades to study and manipulate fluids in microstructures. It has the potential to provide smart microdevices which can change how the modern biology, chemical synthesis, and point-of-care diagnostics are performed. Microfluidics offers many advantages including minute quantities of samples and reagents, compact ability, low cost, rapid, high resolution and sensitive analyses [11]. However, microfluidic-based technologies have not been applied in clinical settings despite decades of advancements in the microfluidics technology. These devices provide valuable information about the bacteria, its resistance and treatment with antibiotics while tackling AMR.

Nevertheless, these devices are not widely accepted in the clinical environment and lack backing from key opinion leaders (KOL) in the field of clinical diagnostics. The chief reasons for lacking clinical acceptability include fabrication and operational complexity, the requirement of resources and sophisticated equipment such as pumps, optics and statistical analysis, running costs and portability. Ideally, the technology to be clinically accepted and deployed for POC diagnostics, especially for AMR testing, must have the following abilities in a single, standalone and portable device. It must be able to detect bacteria, perform AST and determine MIC within few hours, on-site and near the patient. It must be accurate, reliable and reproducible, simple to fabricate, assemble and operate, and cheaper to buy with low maintenance costs as compared to current technologies. It must be scalable and commercially available in the market, and more importantly, the results must be able to guide treatments almost in real-time and save lives. This review mainly focuses on the current developments of microfluidic-based AMR testing devices that have the potential to direct the technology towards the benefit of human health in clinical settings.

\section{MICROFLUIDIC BASED TECHNOLOGIES FOR ANTIMICROBIOTIC RESISTANCE}

Due to the limitations of conventional AMR techniques discussed above, microfluidic-based platforms have been developed to enhance understandings of bacterial resistance, provide smart AST and MIC testing tools, and development of the new drugs. This review aims to summarise the recent developments in microfluidic-based AST and MIC testing tools in different formats of microfluidics technology such as detection of biochemical and physiological changes in bacterial culture [12-14]. For example, Tang et al. [14] developed a microfluidic-based $\mathrm{pH}$ sensor device to detect small changes in $\mathrm{pH}$. These changes were measured by the effective optical thickness of the $\mathrm{pH}$-sensitive hydrogel, which swells under acidic conditions. As bacteria utilises glucose during culture that results in the production of acidic metabolites and hence, changes in $\mathrm{pH}$ occur. Some other techniques involving bacterial detection include but not limited to surface plasmon resonance-based biosensors by detecting refractive index changes of bacteria in culture [12], and optofluidics devices coupled with surface-enhanced Raman scattering spectroscopy to specify differences in clinical isolates [15]. Although these types of studies have the ability to provide sensitive bacterial detections, however, the requirement of sophisticated instrumentation such as miniaturised $\mathrm{pH}$ measurement and Raman scattering prove difficult to be integrated into a portable and straightforward microfluidics device.

Antimicrobial susceptibility testing (AST) is a crucial technology to attain information regarding bacterial resistivity towards drugs and, ultimately, controls the spread of AMR. Several microfluidic-based platforms have proven that AST can provide faster results while guiding clinical decisions of antibiotic use and improving patients' health $[16,17]$. For example, in a microfluidic-based AST and MIC study, morphological changes of single bacterial cells were tracked. Moreover, 189 clinical isolates were studied, and MIC was achieved within 4 hours [18] that was a significant reduction in operation time. In another study carried out with the same platform, an AST of Mycobacterium tuberculosis was achieved in 9 days that is nearly six times reduction in bacterial growth in conventional technologies [19]. However, these results while impressive and achieved in a fraction of time compared with conventional methods but utilised highly sensitive optical detection systems and complex statistical calculations. The prospects of these technologies gaining access to the clinical market depend on the miniaturisation of ultra-sensitive detections and robust statistical data analysis. Rapid and multiplexed AST systems were also developed to study bacterial interactions and drugs responses in parallel on a single device while utilising reduced amounts of reagents and samples, and performing analysis in shorter times [20-21]. Sun et al. [22-23] developed an agar-based cell-on-hydrogel platform to study bacterial interactions with different concentrations of drugs. The authors generated twodimensional gradients in a stacking device and obtained AST results within 2-3 hours. The device in itself was able to multiplex assays on a single platform and gels can be pre- 
a

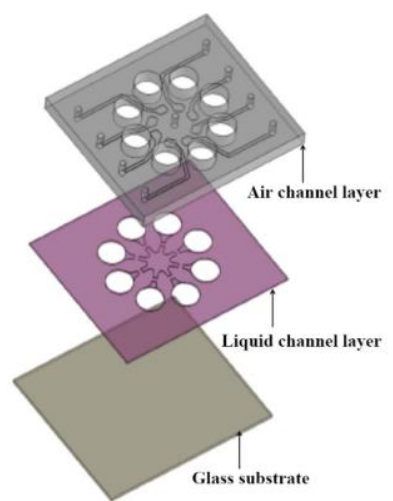

a) An exploded view of the microfluidic chip

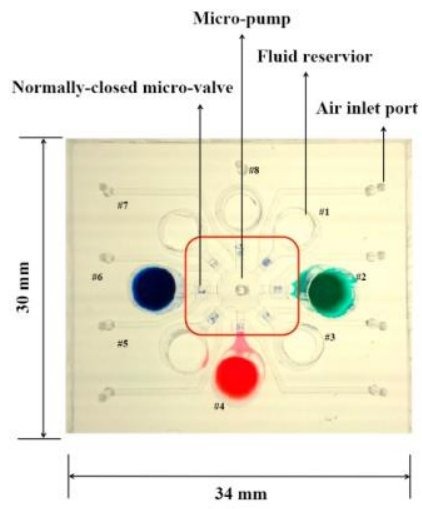

(b) A photograph of the integrated microfluidic chip b

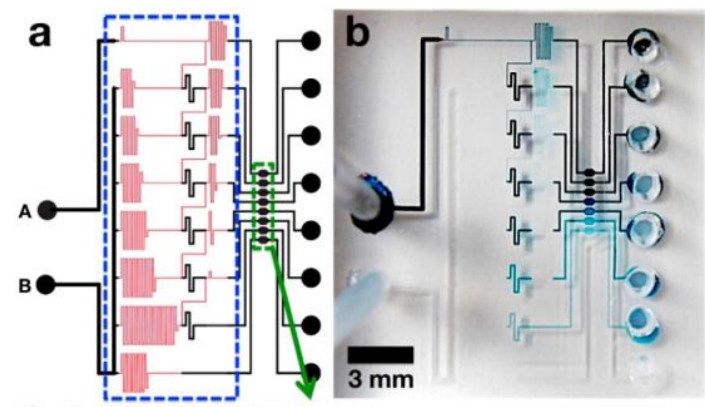

Gradient Generation Cell Culture

C

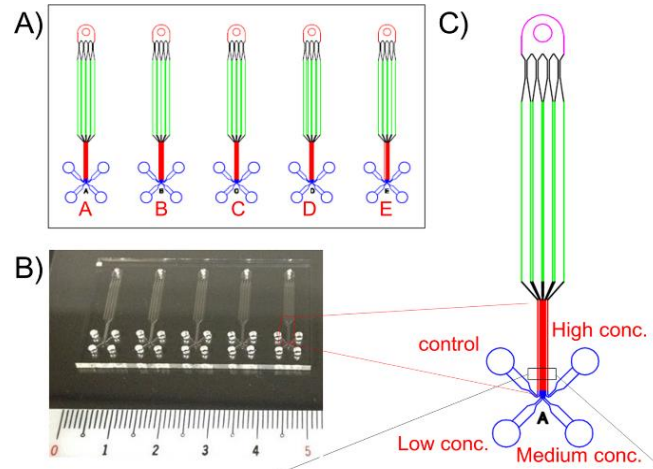

D)

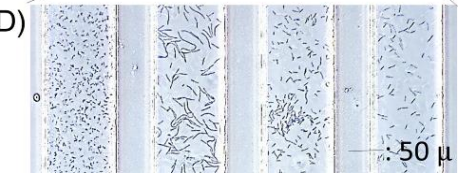

A
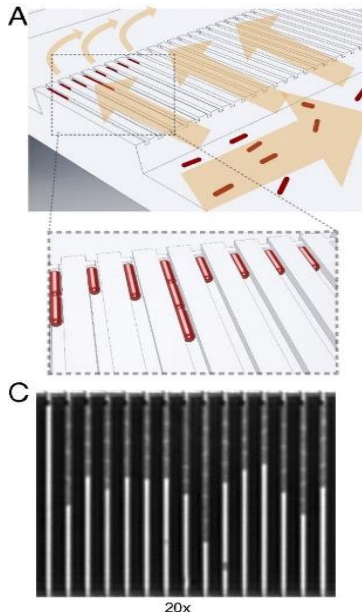

$\mathrm{B}$

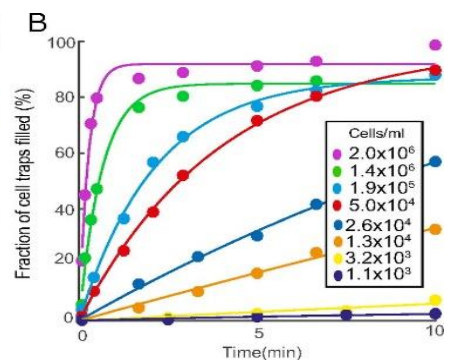

D

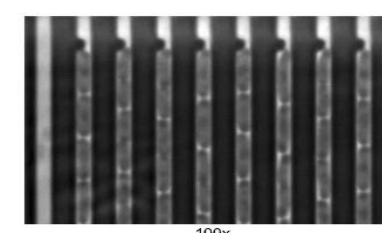

Figure 1: Microfluidic-based devices for rapid AMR detection. a) PDMS/glass microfluidic device consisted of 3 layers namely air and liquid channel layer and glass substrate and eight chambers with built in micro-valves and circular micropumps. The working principle of the device involves opening and closing of the air and vacuum channels and precise control of movement liquid. (Reproduced with permission from [24].) b) A PDMS based device to generate concentration gradients and perform cell culture in eight 30-nanoliter chambers. (Reproduced with permission from [25].) c) Design and photograph of the drug susceptibility microfluidic (DSTM) device for determination of MIC of five drugs, and microscopic image of the growth of $P$ aeruginosa against piperacillin. (Reproduced with permission from [26].) d) Schematic of the bacterial cell tracking of sub- $\mu$ m motions illustrating working principle steps i.e. sample handling and bacterial cell tethering on the wells on glass slide, measurement of motions under microscope, addition of antibiotic concentrations into the wells, and analysis of change in bacterial cell motion to identify antibiotic dose profile. (Reproduced with permission from [27].) e) Design and operation of the AST device based on cell trapping. Rod-shaped bacterial cells are loaded into the device, and fraction of $E$ coli cell trap was measured against time. A microscopic photograph of call capturing regions illustrates trapping of cells at different locations in the device. (Reproduced with permission from [28].)

coated in the channels that offer the possibility of POC diagnostics in healthcare. Broth-based dilution method is a widely used technique in clinical studies to perform ASTs and hence has a great potential to be integrated into microfluidics systems and provide rapid and sensitive ASTs. Several brothbased microdilution methods have been developed such as a microfluidic broth-based dilution method (Fig. 1a) was developed to perform AST and determine MIC [24]. The 
authors performed incubation of vancomycin-resistant Enterococcus cells with five different concentrations of vancomycin in the three-layered PDMS-glass device within few minutes and determined MIC in 24 hours. Sample injection, transport, mixing and dispensing were integrated on an automated platform that provided a flexible, reliable and portable system for clinical applications. The schematic of the integrated microfluidic chip and a photograph illustrating the layers, chambers and working principle of the device are shown in Fig.1a. Another AST microfluidics device (Fig. 1b) based on broth dilutions to generate gradient concentrations and perform cell culture in eight 30-nanoliter chambers was developed by Kim et al. [25]. With $20 \mu \mathrm{L}$ samples of Escherichia Coli (E coli) and, ampicillin or streptomycin were used to determine MIC in three hours. These broth-based studies were able to generate valuable results in shorter times and ideally can proceed for POC diagnostics because of the use of widely accepted cell culture methods. However, these devices require miniaturisation of pumping systems. The currently available pumping modules (the ones used in these studies) cannot perform AMR tests for diagnostics because of the requirement of the expertise in pump handling, and the bulky nature of these systems such as tubing, connections, and manual fluid handlings makes it difficult to deploy for AMR testing. In addition, it increase the costs of disease treatments per patient and hence almost impossible to afford for modern healthcare systems around the world. Therefore, only inexpensive pumping modules and portable, sensitive detections can lead these techniques to the clinical diagnostics for AMR.

Single cell imaging of the bacteria treated with antibiotics can also provide valuable information about the resistivity of the microbes [26-28] such as Yoshimi and co-workers [26] developed drug susceptibility testing microfluidic (DSTM) device to perform AST of Pseudomonas aeruginosa within 3 hours. The device consisted of five sets of microchannels with one inlet and four narrowly fabricated channels for the microscopic view. Bacteria was introduced into the channels precoated with antibiotics and incubated for $3 \mathrm{~h}$ as shown in Fig. 1c. The system was used to achieve 101 isolated strains of bacteria by evaluating the cell numbers and shapes of bacterial cells in different chambers. Different types of drugs reacted differently with the bacteria such as susceptible cells elongated upon incubation with ciprofloxacin, ceftazidime, meropenem and piperacillin while amikacin affected the cell shape minorly. In another bacterial imaging technique, Syal et al. [27] developed an imaging and tracking technology to identify sub- $\mu \mathrm{m}$ bacterial cell motions (Fig.1d). E coli O157:H7 and uropathogenic $E$ coli (UPEC) were placed on a glass surface, and their sub- $\mu \mathrm{m}$ movements were tracked upon treatment with antibiotics. The device simply worked in four steps: (i) Bacterial cells (UPEC) spiked in urine samples were loaded onto the glass slide with wells; (ii) microscope was used to monitor and record bacterial cell motions; (iii) antibiotics were added to see the effect of drugs on bacterial cell motions; and (iv) different antibiotic concentrations were performed on the device. The experiments revealed that antibiotic concentrations reduced movements (Fig 1d). The platform was able to determine MIC in 2 hours, and the results were compared with conventional AST methods. This type of method can be fast, reliable and inexpensive because of the requirement of simple and inexpensive device fabrication, and device operation without the requirement of sophisticated pumping systems. However, this requires sensitive microscopic imaging and robust analysis of bacterial response is required to track the shape and movements of bacterial cells. Recently, Balteken et al. [28] developed an interesting microfluidics platform for testing urinary tract infection (UTI) within 30 minutes by capturing bacterial cells on a microfluidic chip and monitoring their growth via a microscope (Fig. 1e). The authors were able to detect growth rate changes of UPEC bacterial cells upon treatment with nine antibiotics in minutes. Additionally, 49 isolates were found resistant to ciprofloxacin within 10 minutes. This system has the potential to be used as a point-of-care device for testing urinary tract infection and guiding correct treatments at the clinic only and if miniaturised microscopic detections and automatic statistical analysis can be performed portably.

Another unique capability of microfluidics is to manipulate smaller volumes and compartmentalising biomolecules into nano-litre sized droplets. Droplet-based microfluidics is well known for the reduction or elimination of contamination between different samples, faster mixing in droplets, and controlled and reproducible droplet generation with biochemical reactions inside these tiny confined volumes [2931]. Bacterial cells can be injected into droplets (either single or multiple cells) which increases the density of cells per droplet volume and hence, promotes the accumulation of released molecules around the cell. This as a result, reduces the incubation time and provides faster detection of bacterial resistance towards drugs. Droplet-based microfluidics has been used to develop robust and reliable AST testing devices as demonstrated by Aveshar et al. [32]. The authors developed a rapid and scalable AST platform to provide urinary tract infection results within one day. A simple device was constructed to combine nano-litre wells equipped with lyophilised antibiotics with resazurin assay while injecting samples with a standard lab-based pipette (Fig 2a). Firstly, lyophilised antibiotics were placed into the restriction channels, and a $10-\mu \mathrm{L}$ pipette was used to inject bacteria suspension with resazurin and carrier oil. Secondly, the oil plug followed and separated the wells from each other. Finally, a fluorescence signal was monitored in each well. Clinical isolates were directly studied, and the results were compared with standard automated equipment. The authors also demonstrated the ability of the device to multiplex analyses by parallelising nano-litre wells and to equip with lyophilised antibiotics. The device offers promising results and fulfils the needs of simple operating procedures with inexpensive fabrication and multiplexing ability. However, the idea to deposit lyophilised antibiotics in miniaturised wells requires reproducible and reliable deposition in each well which can be difficult and expensive. Furthermore, the use of resazurin requires a fluorescent signal to be detected from these chambers and hence, a miniaturised fluorescence detection system is also required on-board for sensitive detections and analysis.

Droplet-based AMR research studies involve active droplet generation, and encapsulation of bacterial sample and antibiotics into droplet plugs such as Kaushik et al. [33] developed DropFAST, a bacterial growth detector and AST assessor in pico-litre droplets as shown in Fig. 2b. Resazurin based fluorescent assay was used to quantify the effect of gentamycin on growing $E$ coli confined in 20 pico-litre droplets. Bacteria and antibiotic plus viability indicator were injected into the microfluidic chip and droplets were 


\section{a}

A

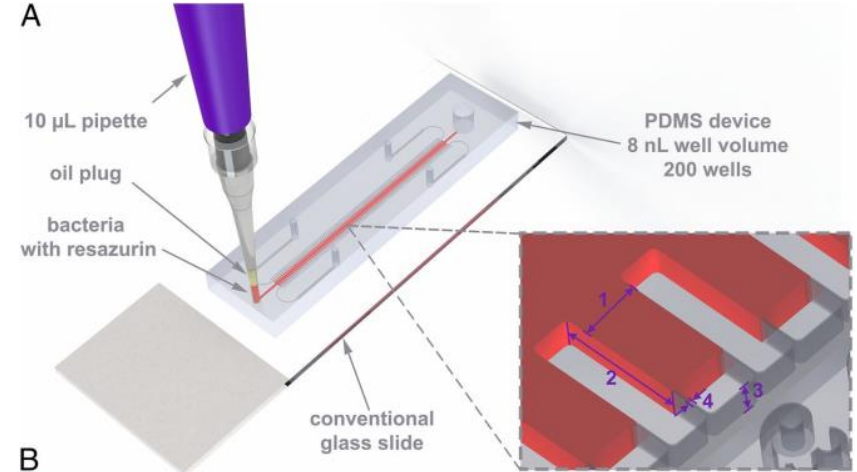

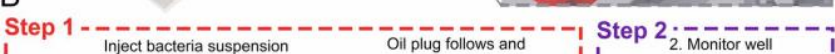

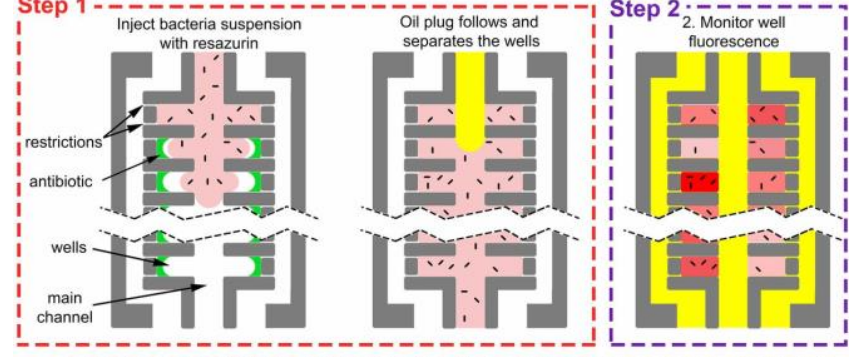

๑ PDMS $\square$ Air $\square$ Antibiotic $\square$ Growth medium with resazurin $\square$ Reduced resazurin $\square$ FC-40 oil b

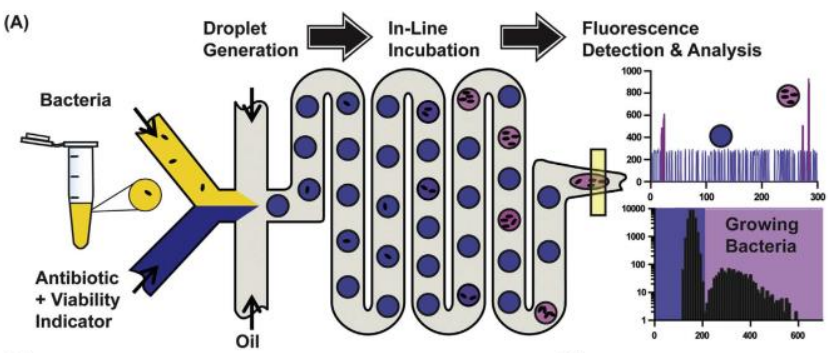

C

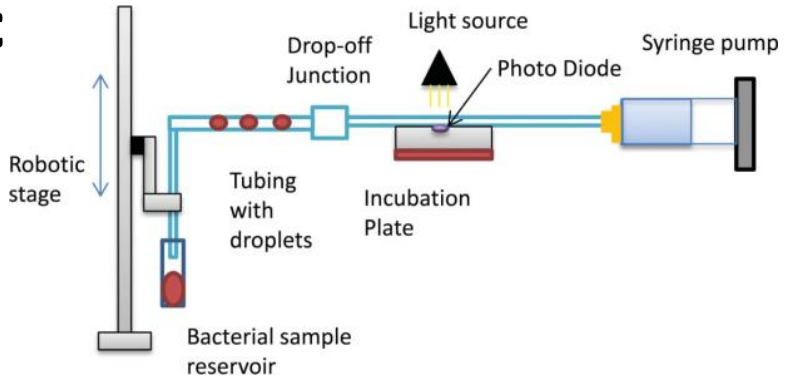

d

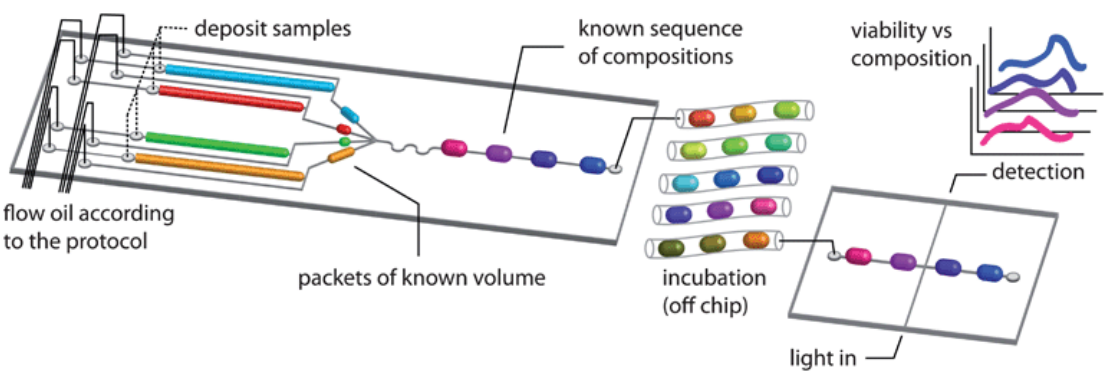

Figure 2: Droplet-based microfluidics devices for rapid detection of bacterial cells in confined droplets. a) Stationary nanoliter droplet array (SNDA) AST device illustrating device design and working principle. Two rows of $8 \mathrm{~nL}$ well prefilled with lyophilized antibiotics are filled with sample followed by carrier oil, breaking into compartmentalized droplets. The architect of device eliminates evaporation, provides oxygen via oil and allows measurement of direct fluorescence from the wells. (Reproduced with permission from [32].) b) DropFAST platform showing bacteria and antibiotic plus viability indicator encapsulation in droplets, incubation and fluorescence detection of resazurin. Histogram fluorescence analysis of droplets with bacteria provides concentration profiles of bacterial presence confined inside droplets. (Reproduced with permission from [33].) c) Schematic of the generation of $E$ coli and antibiotics droplets, and detection of the signal from incubation chambers. Droplets coalesce in incubation chamber to initiate reaction. (Reproduced with permission from [34].) d) Schematic of the droplet generation from different volume sizes for automated AST. Known sequence of compositions of droplets are generated and incubated off-chip and polythene tubing. Droplets are then injected into a channel to detect bacterial metabolism via optical fiber and spectrophotometer. (Reproduced with permission from [35].)

generated. These droplets were incubated in the incubation zone of the microchip and then passed from a narrow $10-\mu \mathrm{m}$ wide channel for fluorescent detection and analysis. This system allows faster droplet generations, and lower incubation times in droplets, however, this format of the microfluidics technology requires sequential injection of different concentrations of antibiotics and multiplexing it would require multiple pumping lines, increasing the cost of the AMR tests per patient. Moreover, the variability of bacterial encapsulation in multiple droplets also poses a challenge for statistical analysis of final concentrations in the sample. Similarly, in stokes laboratories [34], E coli bacteria was cultured in microfluidic droplets (Fig. 2c), and continuous monitoring of individual droplet reaction combinations was performed. The authors detected the lysogene broth culture media for bacterial growth continuously while providing results in $1 \mathrm{~h}$ incubation time. This technology uses droplet on demand technique which generates droplets via a command controlled interface and this is a significant difference between this technology and the DropFAST which uses active droplet generation technique [33]. On the other hand, this study detects the colour change of lysogene broth during incubation while DropFAST detects fluorescence signal. Droplet on demand systems offer more control over the size, speed and flow control of the droplets. Similarly, Churski et al. [35] attempted to automate the MIC determination and $E$ coli toxicity testing against ampicillin, tetracycline and chloramphenicol via generation of multiple droplets on demand. This system utilised less than $100 \mu \mathrm{L}$ of sample and reagent volumes and provided user-programmed bacterial and antibiotic concentrations loaded into the droplets. Furthermore, the droplets were stored in a sequence, incubated and injected into a spectrophotometer for cell growth measurements as shown in Fig. 2d. This format of droplet generation provides an ideal system to automate the AMR analysis with miniaturised droplet detections and has the potential to multiplex the assays in parallel. However, it requires expensive pumping systems such as one available in 
the market known as Mitos Dropix Droplets on Demand by Dolomite Microfluidics. Currently, these type of systems are only available for research purposes and further optimisations and miniaturisations are required before they can be used for POC diagnostics. Human blood serum has also been tested in droplet-based microfluidics such as Boidicker et al. [36] developed a platform to detect antibiotic susceptibility in nano-litre droplet plugs using methicillin-resistant Staphylococcus aereus (MRSA) bacteria against multiple drugs. The authors also detected MIC in single experiments using cefoxitin against $S$ aereus, and the device was proven to be useful for differentiating between sensitive and resistive bacteria in human blood serum. All the above-mentioned droplet-based studies at their current stages provide valuable results which detect bacteria and help understand the bacterial response towards antibiotics but lack the ability to be used for near-patient diagnostics.
Overall, the benefits of droplet-based systems include confinement of cells in droplets, which increases surface area for reaction, less incubation time or even no pre-incubation required. In addition, droplets configuration allows multiplexing drug resistance to be studied in parallel format where different combinations of multiple drug concentrations against bacterial growth can be tested on a single platform. This, in turn, provides measurements in shorter times and can lead to more informed clinical decisions providing an opportunity for the use of narrow-spectrum antibiotics in disease treatments. But the droplet generation methods used in these studies require multiple pumping modules, sensitive fluorescent detections and droplet analysis of millions of droplets which hinders their use for POC diagnostics in healthcare and makes them a suitable technology for laboratory-based research. a

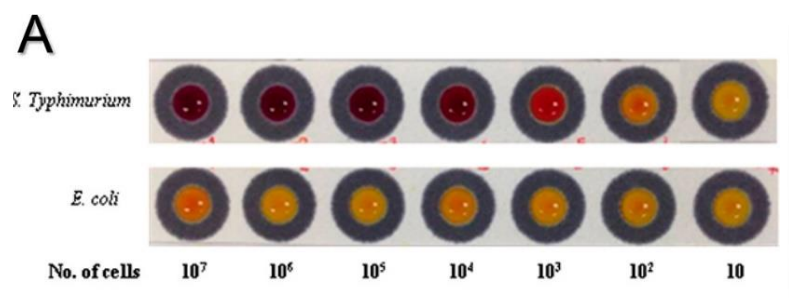

B

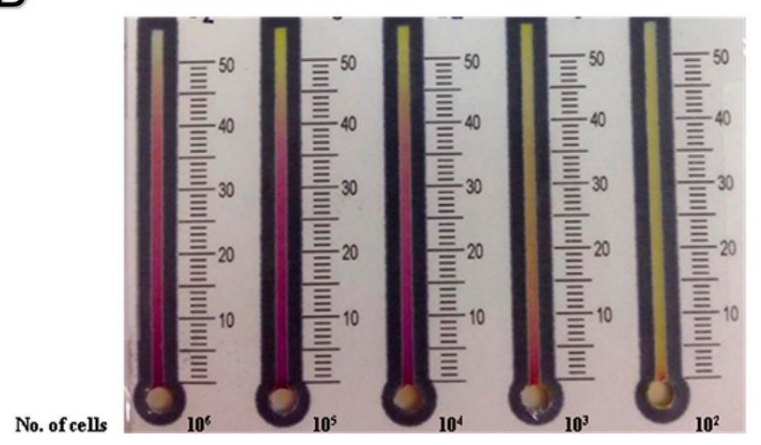

b

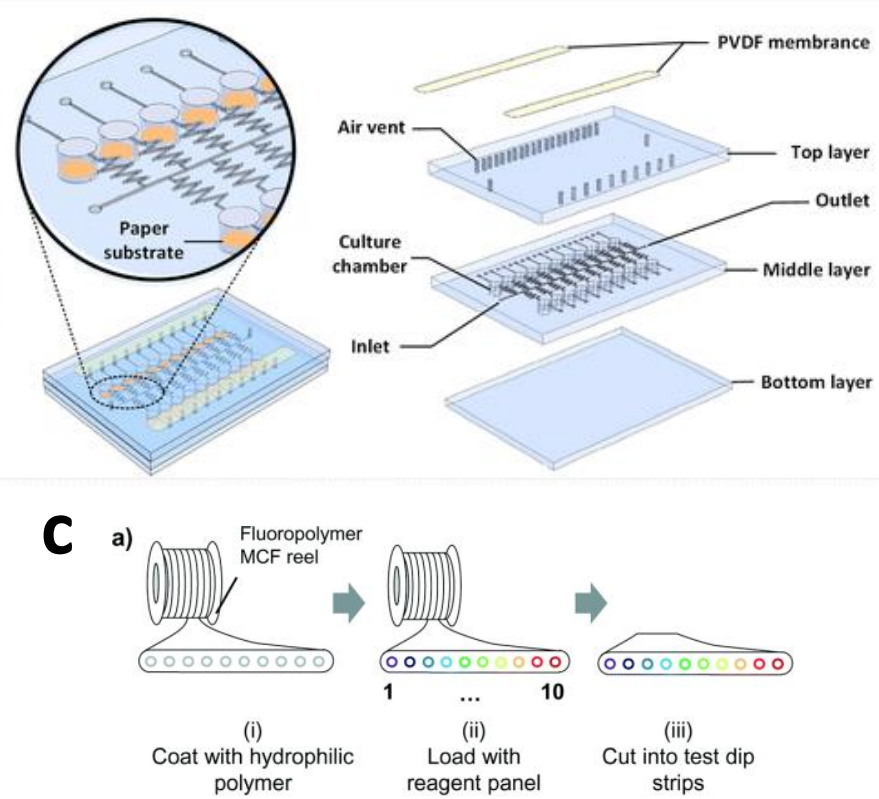

b)

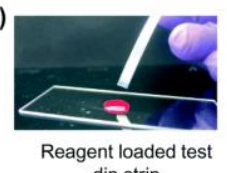

c)

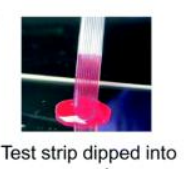
sample

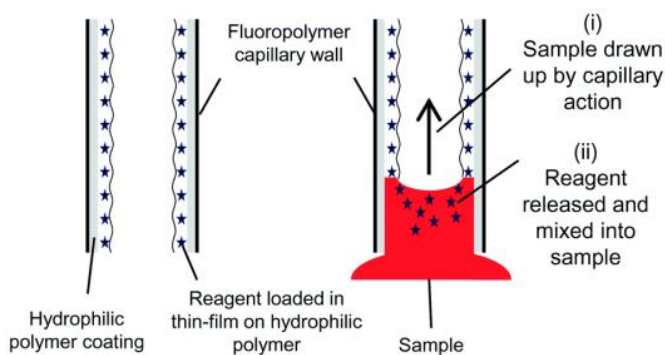

Figure 3: Paper-based and capillary-flow driven microfluidics devices for AMR detection. a). Paper-based analytical device (PAD) to detect Salmonella typhimurium and $E$ coli. Sandwich immunoassay using B-galactosidase and colorimetric assay with chlorophenol red- $\beta$-D-galactopyranoside was performed on paper. The incubation times were optimized on well array devices and chemometers and the bacterial growth was determined measuring the length of the progression on chemometers. (Reproduced with permission from [45].) b) Schematic of the PDMS/paper device for detection of uropathogens on precoated paper substrate inside PDMS channels and wells. The microchip consists of three layers namely top layer with air vents, inlets and outlets, middle layer to connect layers and bottom layer with chambers to insert paper substrates. (Reproduced with permission from [47].) c) Lab-on-a-stick device schematic illustrating FEP test strips, reagent and sample loading into test strips via capillary action. The FEP test strips were precoated with antibiotics and dipped into the sample upon which reagents released into aqueous sample react with bacteria. (Reproduced with permission from [48].) 
As an alternative to active microfluidic-based techniques, paper-based microfluidic devices have become useful due to precise on-paper passive flow control, easy operation, less contamination/evaporation issues, portable and disposable nature, and cost-effectiveness [37, 38]. These types of devices have found important applications in POC and medical diagnostics. Especially the devices are well suited for remote areas where expensive sample handling and sophisticated pumping systems are limited [39]. Several paper-based microfluidics devices detecting AMR have been reported [4047]. Recently, Srisa-Art et al. [45] developed a paper-based analytical device (PAD) to detect Salmonella typhimurium via on-paper sandwich immunoassay using B-galactosidase as enzyme and colourimetric assay bacteria detection with chlorophenol red- $\beta$-D-galactopyranoside (Fig. 3a). The authors also tested bird faeces for bacterial detection without any interferences from other pathogenic bacteria where the detection was achieved measuring the length of the coloured band on the paper making it suitable for machine free testing. This technology provides a platform to perform cheaper AMR assays, but the sensitivity of the assay was found to be lower due to the matrix effect in the samples. Nevertheless, this format of paper-based technology can be highly regarded as a cheaper alternative to active microfluidics devices and will get through the commercial challenges once fully optimised. In another paper-based AMR device, Katherine et al. [46] developed a PAD for detecting $\beta$-lactamase resistance from the environment such as water. In this study, the authors investigated the bacterial species isolated from water in arrays of fabricated wells on paper and detected via smartphone image analysis. This type of testing can prevent spread of resistant bacteria to the environment at a mere cost and save expensive transportation of water samples to central-labs and laborious testing. To implement this study for POC diagnostics, complete miniaturisation of instrumental tools and extensive research is required including optimization of complex samples such as blood, urine or milk. In another study done by Xu et al. [47] one-step bacteria identification and AST testing were achieved on PDMS/Paper-based device. The device consisted of chambers and sample inlet channel made of PDMS and a paper preloaded with antimicrobial agents and chromogenic substrate (Fig. 3b). Multiple uropathogens were detected on the device for UTI disease via detection of colour changes from the chromogenic reaction in chambers. Detection of three different bacteria on a single device and MIC determination was achieved within 15 hours, which is much quicker than standard methods (3-4 days). This device reduced a significant amount of time for UTI tests and potentially can reduce the cost of tests per patient paving the way for deployment in clinical settings. However, it must fully satisfy commercialisation needs such as requirement of portability, detection and assay reproducibility. Generally, paper-based devices are simple, cheaper, easy to fabricate and more importantly are less reliant on sophisticated instruments for operational procedures. Therefore, significant research is required to overcome technical challenges faced by paperbased POC devices, especially in combating AMR in healthcare.

Fluids can also be driven in polymer/glass microfluidics devices by capillary action known as capillary-driven flow microfluidics that eliminates the need for external pumping systems. These types of devices can easily be operated as the reagents/antibiotics are dry stored in the microchannels where samples are driven into the microchannels by directly placing devices into the sample fluids. Many such devices have been developed and reported for diagnostics applications [48-51]. Reis et al. [48] developed fluoropolymer-based (FEP) lab-ona-stick test strips to study multi-analyte assays in shorter times (Fig. 3c). By dipping reagent stored test strips into sample liquids, it allowed the liquid to travel via capillary action. Following incubation, the individual test strip (with ten channels) was read in a custom-built scanner. In this way, the device was tested for $\mathrm{ABO}$ blood typing, identification of three different bacterial cells and MIC determination using multiple drugs. Although these type of capillary-driven flow devices are immature at this stage, further improvements to these systems can provide a new generation of microfluidics diagnostic tools that can be more sensitive, cost-effective and portable for the global fight against AMR spread. Ideally, capillary-driven flow devices are suited for POC diagnostics because this type of microfluidics has the potential to provide rapid, inexpensive and simple clinical assays in shorter times and near the patient. The reagents can be pre-coated on to the surface of the channels and by merely dipping the device into the sample from patients, incubating and getting results within few hours can lead to the development of the simple POC devices accepted by the clinical environment.

Indeed, there is a global requirement for development of handheld devices to identify different types of bacteria and determine MIC for effective treatments. Current microfluidics approaches (some mentioned in this paper) provide solutions for running assays in smaller microfluidics devices yet require bulky optical detection systems to analyse these results. This, as a result, is limiting the portability and wider acceptability of these devices. An alternative to these bulky optical devices, a smartphone can be used for diagnostics purposes where a mobile phone can be converted into a mini microscope or spectrophotometer while data processing programs can be installed on cell-phones to provide automated results. Several studies have been performed using a smartphone as a microscope or optical detector, such as used for ELISA assay detection in microchip [52] and quantitation of prostatespecific antigen (PSA) in FEP test strips [53]. Recently, Barbosa et al. [53] developed a smartphone-based detection system to quantify PSA using enzymatic amplification and colourimetric assay substrate reaction (Fig. 4a). A magnifying lens and light source was attached to the mobile phone camera to detect the colourimetric signal from the FEP test strips. The system was also adapted to detect fluorescent signal of the assay by adding dichroic mirror and a background UV black light. This smartphone-based detection developed in this study has a great potential to be combined with paper-based or capillary-driven flow microfluidics to yield an ever demanded AMR testing system for deployment in clinical settings. Furthermore, Steve et al. [54] 3D printed an attachment consisting of an array of LEDs to illuminate microtiter plates and optical fibre to capture light from the microwells on the titer plate (Fig. 4b). The smartphone was able to measure the turbidity within $1 \mathrm{~min}$. Clinical isolates of Klebsiella pneumoniae in 78 patients were tested against 17 antibiotics with this smartphone-based device, and the AST accuracy was within the FDA defined criteria, i.e. turbidity 
a
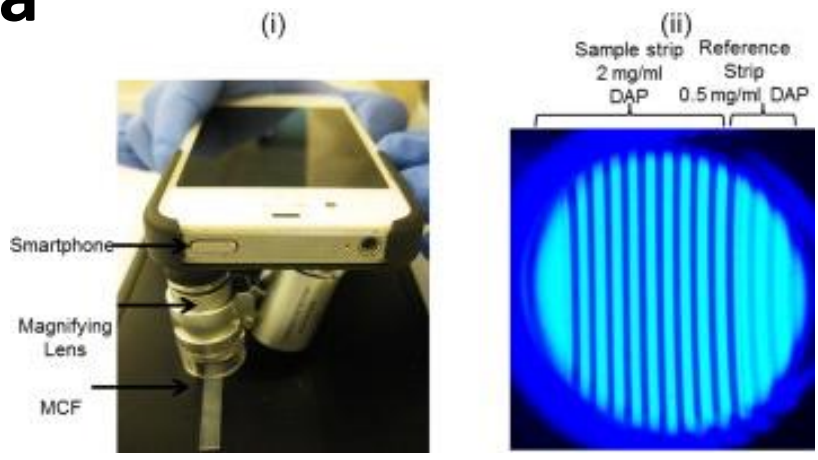

C

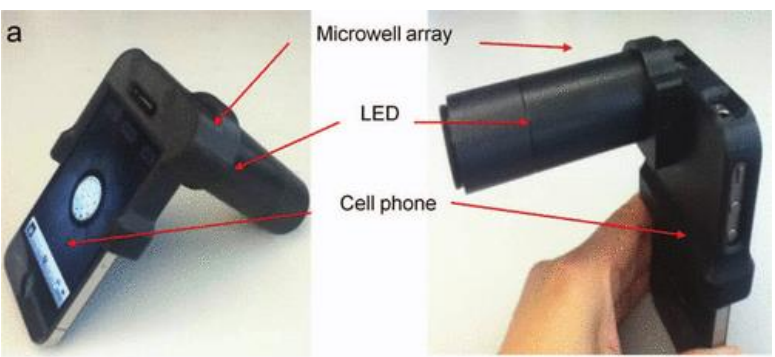

b
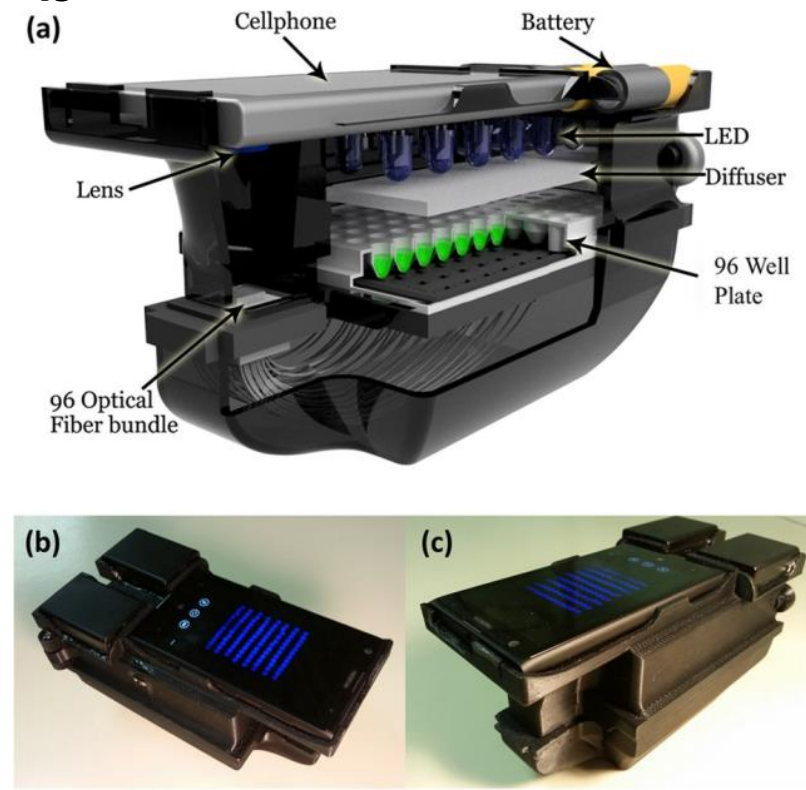

(c)

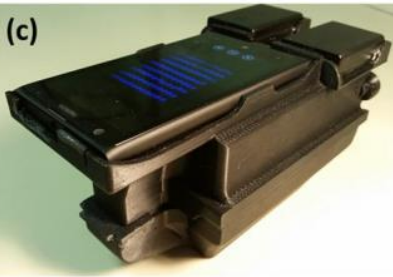

Figure 4: Smartphone-based portable devices for POC diagnostics. a) A photograph of a smartphone-based detection system to quantify PSA using enzymatic amplification and colorimetric assay substrate. A magnifying lens and light source were attached to the mobile phone camera to detect colorimetric signal from the FEP test strips and a reference channel was used to normalize results for each channel. The system was also adapted to detect fluorescent signal by adding dichroic mirror and a background UV black light. (Reproduced with permission from [53].) b) 3D illustration of the Portable 96-well microplate system for AST. A 3D printed attached was fabricated including LED, diffuser and optical fiber for light illumination. The signal of the sample was collected via mobile phones camera. (Reproduced with permission from [54].) c) Photographs of rapid AST testing cell phone-based spectrophotometric device Bacterial growth was detected in microwell arrays using smartphone's camera. (Reproduced with permission from [55].)

measurement (98.21\%), MIC (95.12\%) and AST (99.23\%). These results while encouraging were demonstrated using a 96 well plate and require time-consuming sample handling steps. Therefore, the powerful detection capabilities of smartphone must also be combined with miniaturised AMR testing techniques already developed to gain fruitful outcome for commercialisation of these tools. In contrast, Kadlec et al. [55] developed a smartphone-based spectrophotometric system for detecting the bacterial growth in microwell arrays (Fig. 4c). The authors pre-coated the walls of the gas permeable wells with antibiotics, cultured bacteria with colourimetric viability substrate and read the signal from a smartphone camera. Using this system, AST profiling of cultured bacteria and uropathogens for UTI was performed. The system was fully miniaturised for UTI testing and can lead towards the POC diagnostics in healthcare. However, further improvements to simplify sample handling and multiplexing in parallel should be exploited to gain access to the clinical diagnostics market. Contrary to optical detections via smartphone, an electrochemical detection method [56] and colourimetric scanning [48] have also been attempted for bacterial identification to build standalone and portable AMR testing tools. Nonetheless, these tools are immature at this stage and require the development of cost-effective and reliable methods to overcome the dilemma of gaining trust from key opinion leaders in clinical diagnostics.

\section{CONCLUDING REMARKS}

Current conventional AMR detection technologies provide time-consuming, costly, labour-intensive and central lab-based solutions, limiting their applications. Microfluidics has been developed for decades, and the technology has emerged as a powerful tool for point-of-care (POC) diagnostics of AMR in healthcare providing, simple, robust, cost-effective and portable diagnostics. Various microfluidics devices have been developed to achieve this goal. Miniaturisation and integration of different tools have been attempted to produce handheld or standalone devices for rapid AMR testing. The successes have been registered in research articles; however, the potentials of microfluidics technology in tackling AMR have not been fully achieved in clinical settings. This article reviews some of the most popular and important research studies recorded in the literature.

Microfluidics has not gained confidence in the clinical environment and has not made a breakthrough in the commercial industry worldwide. This is mainly due to the nature of delicate microfluidics innovations and requirement of expertise and sophisticated instruments for commercialisation of these devices. The surface properties of materials require expensive treatments, and hence reproducibility issues can arise from this problem. There is a lack of simplicity and ease of use of microfluidic devices along with scalability issues that have made these microfluidics devices less favourable commercially. Although, there have been multiple research studies (some mentioned above) which provide solutions to tackle AMR, yet no microfluidic-based systems are combating AMR in the field. Therefore, simple, affordable and rapid systems are still a requirement to bridge the gap between research and fielddeployable POC diagnostics [57]. Following research in AMR tackling, must be able to provide simple, inexpensive and fully portable systems. Current research studies reviewed in this paper provide a clear view that the focus of the research in the last decade has been miniaturising current assay techniques to 
provide rapid AMR testing. However, all the characteristics required by clinicians such as simplicity, cost-effectiveness, portability, accuracy and time-efficiency are not found in a single device. This paper provides a platform to compare different techniques and match suitable technologies to develop new devices which include all the characteristics mentioned above. One of the best and emerging scenario is combining paper-based or capillary-driven flow microfluidics with smartphone-based detection which can be cost-effective, simple and portable. Ideally, these devices are cheaper to fabricate and simple to operate such as pre-coated testing strips, or channels can be dipped in the sample, and the colour development or signal can be monitored via smartphone. The smartphone must be able to analyse the signal, and the results must be provided within a few hours while the patient still stays in the clinic so that onspot and well-informed clinical decisions can be made for the better treatment of the patients.

\section{CONFLICT OF INTEREST}

The authors confirm that this article content has no conflict of interest.

\section{ACKNOWLEDGEMENTS}

We thank the funding from the Economic and Social Research Council UK (ES/S000208/1).

\section{REFERENCES}

[1] O’Neill, J. Rapid Diagnostics: Stopping Unnecessary Use of Antibiotics. Review on Antimicrobial Resistance., Welcome Trust and HM Government, 2015.

[2] Smith, R.; Coast, J. The true cost of antimicrobial resistance. BMJ., 2013, 346, f1493.

[3] Aarestrup, F. Sustainable farming: get pigs off antibiotics. Nature., 2012, 486, 465-466.

[4] Lazcka, O.; Del Campo, F. J.; Muñ oz, F.X. Pathogen detection: a perspective of traditional methods and biosensors. Biosens. Bioelectron., 2007, 22, 1205-1217.

[5] Aroonnual, A.; Janvilisri, T.; Ounjai, P.; Chankhamhaengdecha, S. Microfluidics: innovative approaches for rapid diagnosis of antibiotic-resistant bacteria. Essays in Biochemistry., 2017, 61, 91101.

[6] Pulido, M. R.; García-Quintanilla, M.; Martín-Peña, R.; Cisneros, J. M.; McConnell, M. J. Progress on the development of rapid methods for antimicrobial susceptibility testing. J. Antimicrob. Chemother., 2013, 68, 2710-2717.

[7] Schofield, C.; B. Updating antimicrobial susceptibility testing methods. Clin. Lab. Sci., 2012, 25, 233-239.

[8] Didelot, X.; Bowden, R.; Wilson, D. J.; Peto, T. E. A.; Crook, D. W. Transforming clinical microbiology with bacterial genome sequencing. Nat. Rev. Genet., 2012, 13, 601-612.

[9] Hrabak, J.; Chudackova, E.; Walkova, R. Matrix-assisted laser desorption ionization- time of flight (MALDITOF) mass spectrometry for detection of antibiotic resistance mechanisms: from research to routine diagnosis. Clin. Microbiol. Rev., 2013, 26, 103114

[10] Kinnunen, P.; McNaughton, B. H.; Albertson, T.; Sinn, I.; Mofakham, S.; Elbez, R.; Newton, D. W.; Hunt, A.; Kopelman, R. Self-assembled magnetic bead biosensor for measuring bacterial growth and antimicrobial susceptibility testing. Small., 2012, 8 , $2477-2482$.

[11] Whitesides, G.; M. The origins and the future of microfluidics. Nature., 2006, 442, 368-373.

[12] Chiang, Y. L.; Lin, C. H.; Yen, M. Y.; Su, Y. D.; Chen, S. J.; Chen, H. F. Innovative antimicrobial susceptibility testing method using surface plasmon resonance. Biosens. Bioelectron., 2009, 24, 19051910.

[13] Karasinski, J.; White, L.; Zhang, Y.; Wang, E.; Andreescu, S.; Sadik, O. A.; Lavine, B. K.; Vora, M. Detection and identification of bacteria using antibiotic susceptibility and a multi-array electrochemical sensor with pattern recognition. Biosens. Bioelectron., 2007, 22, 2643-2649.

[14] Tang, Y.; Zhen, L.; Liu, J.; Wu, J. Rapid antibiotic susceptibility testing in a microfluidic pH sensor. Anal. Chem., 2013, 85, 27872794

[15] Lu, X.; Samuelson, D. R.; Xu, Y.; Zhang, H.; Wang, S.; Rasco, B. A.; Xu, J.; Konke, M. E. Detecting and tracking nosocomial methicillin-resistant Staphylococcus aureus using a microfluidic SERS biosensor. Anal. Chem., 2013, 85, 2320-2327.

[16] Bauer, K. A.; Perez, K. K.; Forrest, G. N.; Goff, D. A. Review of rapid diagnostic tests used by antimicrobial stewardship programs. Clin. Infect. Dis., 2014, 59, S134-S145.

[17] Kerremans, J. J.; Verboom, P.; Stijnen, T.; Hakkaart-van, R. L.; Goessens. W.; Verbrugh, H. A.; Vos, M. C. Rapid identification and antimicrobial susceptibility testing reduce antibiotic use and accelerate pathogen-directed antibiotic use. J. Antimicrob. Chemother., 2008, 61, 428-435.

[18] Choi, J.; Yoo, J.; Lee, M.; Kim, E. G.; Lee, J. S.; Lee, S.; Joo, S. Song, S. H.; Kim, E. C.; Lee, J. C.; Kim, H. C.; Jung, Y. G.; Kwon, S. A rapid antimicrobial susceptibility test based on single-cell morphological analysis. Sci. Transl. Med., 2014, 6, 267ra174.

[19] Choi, J.; Yoo, J.; Kim, K. J,; Kim, E. G.; Park, K. O.; Kim, H.; Kim, H.; Jung, H.; Kim, T,; Choi, M.; Kim, H. C.; Ryoo, S.; Jung, Y. G. Kwon, S. Rapid drug susceptibility test of Mycobacterium tuberculosis using microscopic time-lapse imaging in an agarose matrix. Appl Microbiol Biotechnol., 2016, 100, 2355.

[20] Mohan, R.; Mukherjee, A.; Sevgen, S. E.; Sanpitakseree, C.; Lee, J.; Schroeder, Paul, C. M.; Kenis, J. A. A multiplexed microfluidic platform for rapid antibiotic susceptibility testing. Biosensors and Bioelectronics., 2013, 49, 118-125.

[21] Mohan, R.; Sanpitakseree, C.; Desai, A. V.; Sevgen, S. E.; Schroedera, C. M.; Paul, J. A. Kenis. A microfluidic approach to study the effect of bacterial interactions on antimicrobial susceptibility in polymicrobial cultures. RSC Adv., 2015, 5, 3521135223 .

[22] Sun, H.; Liu, Z.; Hua, C.; Ren, K. Cell-on-hydrogel platform made of agar and alginate for rapid, low-cost, multidimensional test of antimicrobial susceptibility. Lab Chip., 2016, 16, 3130-3138.

[23] Liu, Z.; Sun, Han.; Ren, Dr. K. A Multiplexed, Gradient- Based, Full- Hydrogel Microfluidic Platform for Rapid, High- Throughput Antimicrobial Susceptibility Testing. ChemPlusChem., 2017, 82, 792.

[24] Lee, W. B.; Fu, C. Y.; Chang, W. H.; You, H. L.; Wang, C.H.; Lee, M. S.; Lee, G. B. A microfluidic device for antimicrobial susceptibility testing based on a broth dilution method. Biosensors and Bioelectronics., 2017, 87, 669-678.

[25] Kim, S C.; Cestellos-Blanco, S.; Inoue, K.; Zare, R. N. Miniaturized Antimicrobial Susceptibility Test by Combining Concentration Gradient Generation and Rapid Cell Culturing. Antibiotics., 2015, 4, 455-466.

[26] Matsumoto, Y.; Sakakihara, S.; Grushnikov, A.; Kikuchi, K.; Noji, H.; Yamaguchi, A.; Iino, R.; Yagi, Y.; Nishino, K. A Microfluidic Channel Method for Rapid Drug Susceptibility Testing of Pseudomonas aeruginosa. PLoSONE., 2016, 11, e0148797.

[27] Syal, K.; Shen, S.; Yang, Y.; Wang, S.; Haydel, S. E.; Tao, N. Rapid Antibiotic Susceptibility Testing of Uropathogenic E. coli by Tracking Submicron Scale Motion of Single Bacterial Cells. ACS Sens., 2017, 2, 1231-1239.

[28] Baltekin, O.; Boucharin, A.; Tano, E.; Andersson, D. I.; Elf, J. Antibiotic susceptibility testing in less than 30 min using direct single-cell imaging, PNAS., 2017, 34, 9170-9175.

[29] Hassan, S.; Nightingale, A. M.; Niu, X. Continuous measurement of enzymatic kinetics in droplet flow for point-of-care monitoring. Analyst., 2016, 141, 3266-3273.

[30] Theberge, A. B.; Courtois, F.; Schaerli, Y.; Fischlechner, M.; Abell, C.; Hollfelder, F.; Huck, W. T. S. Microdroplets in Microfluidics: An Evolving Platform for Discoveries in Chemistry and Biology. Angew. Chem., Int. Ed., 2010, 49, 5846-5868.

[31] Schaerli, Y.; Hollfelder, F. The potential of microfluidic water-in-oil droplets in experimental biology. Mol. BioSyst., 2009, 5, 1392-1404.

[32] Avesar, J.; Rosenfeld, D.; Truman-Rosentsvit, M.; Ben-Arye, T.; Geffen, Y.; Bercovici, M.; Levenberg, S. Rapid phenotypic antimicrobial susceptibility testing using nanoliter arrays. PNAS., June 26, 2017, https://doi.org/10.1073/pnas.1703736114.

[33] Kaushika, A. M.; Hsieha, k.; Chena, L.; Shina, D. J.; Liaob, j. C. Wang, T. Accelerating bacterial growth detection and antimicrobial susceptibility assessment in integrated picoliter droplet platform. Biosensors and Bioelectronics., 2017, 97, 260-266. 
[34] Keays, M. C.; O'Brien, M.; Hussain, A.; Kiely, P. A.; Dalton, T. Rapid identification of antibiotic resistance using droplet microfluidics, Bioengineered., 2016, 7, 79-87.

[35] Churski, K.; Kaminski, T. S.; Jakiela, S.; Kamysz, W.; BaranskaRybak, W.; Weibeld, D. B.; Garstecki, P. Rapid screening of antibiotic toxicity in an automated microdroplet system. Lab Chip., 2012, 12, 1629.

[36] Boedicker, J. Q.; Li, L.; Kline, T. R.; Ismagilov, R. F. Detecting bacteria and determining their susceptibility to antibiotics by stochastic confinement in nanoliter droplets using plug-based microfluidics. Lab Chip., 2008,8, 1265-1272.

[37] Mettakoonpitak, J.; Boehle, K.; Nantaphol, S.; Teengam, P.; Adkins, J. A.; Srisa-Art, M.; Henry, C. S. Electrochemistry on Paper- based Analytical Devices: A Review. Electroanalysis., 2016, 28, 1420-1436.

[38] Yang, Y. Y.; Noviana, E.; Nguyen, M. P.; Geiss, B. J.; Dandy, D. S.; Henry, C. S. Paper-Based Microfluidic Devices: Emerging Themes and Applications. Anal. Chem., 2017, 89, 71-91.

[39] Choi, J. R.; Tang, R.; Wang, S.; Wan Abas, W. A.; PingguanMurphy, B.; Xu, F. Paper-based sample-to-answer molecular diagnostic platform for point-of-care diagnostics. Biosens. Bioelectron., 2015, 74, 427-439.

[40] Funes-Huacca, M.; Wu, A.; Szepesvari, E.; Rajendran, P.; KwanWong, N.; Razgulin, A.; Shen, Y.; Kagira, J.; Campbell, R.; Derda, R., Portable self-contained cultures for phage and bacteria made of paper and tape. Lab Chip., 2012, 12, 4269-4278.

[41] Li, C.-z.; Vandenberg, K.; Prabhulkar, S.; Zhu, X.; Schneper, L.; Methee, K.; Rosser, C. J.; Almeide, E. Paper based point-of-care testing disc for multiplex whole cell bacteria analysis. Biosens. Bioelectron., 2011, 26, 4342-4348.

[42] Adkins, J. A.; Boehle, K.; Friend, C.; Chamberlain, B.; Bisha, B.; Henry, C. S. Colorimetric and Electrochemical Bacteria Detection Using Printed Paper- and Transparency-Based Analytic Devices. Anal. Chem., 2017, 89, 3613-3621.

[43] Park, T. S.; Li, W.; McCracken, K. E.; Yoon, J.-Y. Smartphone quantifies Salmonella from paper microfluidics. Lab Chip., 2013, $13,4832-4840$

[44] Jokerst, J. C.; Adkins, J. A.; Bisha, B.; Mentele, M. M.; Goodridge, L. D.; Henry, C. S. Development of a Paper-Based Analytical Device for Colorimetric Detection of Select Foodborne Pathogens. Anal. Chem., 2012, 84, 2900-2907.

[45] Srisa-Art, M.; Boehle, K. E.; Geiss, B. J.; Henry, C. S. Highly Sensitive Detection of Salmonella typhimurium Using a
Colorimetric Paper-Based Analytical Device Coupled with Immunomagnetic Separation. Anal. Chem., 2018, 90, 1035-1043.

[46] Boehle, K. E.; Gilliand, J.; Wheeldon, C. R.; Holder, A.; Adkins, J. A.; Geiss, B. J.; Ryan, E. P.; Henry, C. S. Utilizing Paper-Based Devices for Antimicrobial-Resistant Bacteria Detection, Angew.Chem., Int. Ed., 2017, 56, 6886-6890.

[47] Xu, B.; Du, Y.; Lin, J.; Qi, M.; Shu, B.; Wen, X.; Liang, G.; Chen, B.; Liu, D. Simultaneous Identification and Antimicrobial Susceptibility Testing of Multiple Uropathogens on a Microfluidic Chip with Paper-Supported Cell Culture Arrays, Anal. Chem., 2016 88, 11593-11600.

[48] Reis, N. M.; Pivetal, J.; Loo-Zazueta, A. L.; Barrosb, J. M. S.; Edwards, A. D. Lab on a stick: multi-analyte cellular assays in a microfluidic dipstick. Lab Chip., 2016,16, 2891.

[49] Gervais, L.; Delamarche, E. Toward one-step point-of-care immunodiagnostics using capillary-driven microfluidics and PDMS substrates. Lab Chip., 2009, 9, 3330-3337.

[50] Walker G. M.; Beebe, D. J. A passive pumping method for microfluidic devices. Lab Chip., 2002, 2, 131-134.

[51] Desai, D.; Wu, G.; Zaman, M. H. Tackling HIV through robust diagnostics in the developing world: current status and future opportunities. Lab Chip., 2011, 11, 194-211.

[52] Tseng, D.; Mudanyali, O.; Oztoprak, C.; Isikman, S. O.; Sencan, I Yaglidere, O.; Ozcan, A. Lensfree Microscopy on a Cellphone. Lab Chip., 2010, 10, 1787-1792.

[53] Barbosa, A. I.; Gehlot, P.; Sidapra, K.; Edwards. A. D.; Reis, N. M Portable smartphone quantitation of prostate specific antigen (PSA) in a fluoropolymer microfluidic device. Biosensors and Bioelectronics., 2015, 70, 5-14.

[54] Feng, S.; Tseng, D.; Carlo, D. D.; Garner, O. B.; Ozcan, A. Highthroughput and automated diagnosis of antimicrobial resistance using a cost-effective cellphone-based micro-plate reader. Scientific Reports., 2016, 6, Article number: 39203.

[55] Kadlec, M. W.; You, D.; Liao, J. C.; Wong, P. K. A Cell PhoneBased Microphotometric System for Rapid Antimicrobial Susceptibility Testing. Journal of Laboratory Automation., 2014, 19, $258-266$.

[56] Altintas, Z.; Akgun, M.; Kokturk, G.; Uludag, Y. A fully automated microfluidic-based electrochemical sensor for real-time bacteria detection. Biosensors and Bioelectronics., 2018, 100, 541-548.

[57] Liu, Z.; Banaei, N.; Ren, K. Microfluidics for Combating Antimicrobial Resistance. Trends in Biotechnology., 2017, 35, 12, 1129-1139. 